\title{
BMJ Open Environmental exposure to triclosan and polycystic ovary syndrome: a cross- sectional study in China
}

\author{
Jiangfeng Ye, ${ }^{1}$ Wenting Zhu, ${ }^{2}$ Han Liu, ${ }^{3}$ Yuchan Mao, ${ }^{4}$ Fan Jin, ${ }^{2}$ Jun Zhang ${ }^{3,5}$
}

To cite: Ye J, Zhu W, Liu H, et al. Environmental exposure to triclosan and polycystic ovary syndrome: a cross-sectional study in China. BMJ Open 2018;8:e019707. doi:10.1136/ bmjopen-2017-019707

- Prepublication history and additional material for this paper are available online. To view these files, please visit the journal online (http://dx.doi. org/10.1136/bmjopen-2017019707).

Received 21 September 2017 Revised 6 June 2018 Accepted 18 September 2018

A) Check for updates

(C) Author(s) (or their employer(s)) 2018. Re-use permitted under CC BY-NC. No commercial re-use. See rights and permissions. Published by BMJ.

For numbered affiliations see end of article.

\section{Correspondence to}

Dr Fan Jin; jinfan@zju.edu.cn and Dr Jun Zhang;

junjimzhang@sina.com

\section{ABSTRACT}

Objectives Polycystic ovary syndrome (PCOS) is the most common endocrinopathy of women at reproductive age. Although the aetiology of PCOS remains unclear, potential effects of environmental endocrine-disrupting compounds on the development of PCOS have drawn increasing attention. The aim of the current study was to examine the association between triclosan (TCS) and PCOS, and explore possible mechanisms on how TCS may contribute to the development of clinical manifestations of PCOS.

Design Cross-sectional study.

Setting This study was conducted in one tertiary-level hospital located in Zhejiang, China.

Participants A total of 674 infertile women at 18-45 years of age were recruited in 2014-2015. Participants with $(n=84)$ and without $(n=212)$ PCOS with urinary TCS concentration available were included in the analyses. Methods Urinary TCS concentration was measured using a high-performance liquid chromatography-electrospray ionisation tandem mass spectrometry. Logistic regression model was used to examine the association between TCS and PCOS. Fractional polynomial regression models were built to fit the potential non-linear relationship between TCS concentrations and luteinising hormone (LH) and LH/ follicle stimulate hormone (FSH).

Results The PCOS group had significantly higher level of TCS concentration than the non-PCOS group (the median of TCS (IQR), $\mu \mathrm{g} / \mathrm{g}$ creatinine: $1.49(0.68-3.80)$ vs 1.06 (0.52-3.02), $p=0.0407)$. Compared with the lowest tertile, the highest tertile of TCS concentration was associated with an increased odd of PCOS (OR 2.12, 95\% Cl 1.12 to 3.99). After adjusting for potential confounders, the significant association remained $(\mathrm{OR} 1.99,95 \% \mathrm{Cl} 1.05$ to 3.79). Positive relationships were found between TCS levels and $\mathrm{LH}$ and $\mathrm{LH} / \mathrm{FSH}$ ratio in non-PCOS participants. Conclusions TCS exposure at a relatively low level is associated with PCOS in Chinese women. Further epidemiological studies are needed to confirm our finding, which may have important public health implications.

\section{INTRODUCTION}

Polycystic ovary syndrome (PCOS) is the most common endocrinopathy of women at reproductive age, which encompasses a broad range of signs including hyperandrogenism, polycystic ovaries, oligo-ovulation or anovulation, insulin resistance and elevated luteinising hormone (LH) level. ${ }^{1}$ The latter

\section{Strengths and limitations of this study}

The flexibility of the fractional polynomial model in fitting non-linear relationships between triclosan (TCS) concentrations and luteinising hormone (LH) and LH/follicle stimulate hormone (FSH) in non-polycystic ovary syndrome (PCOS) participants at relatively low levels of TCS exposure is a strength of this study.

- TCS level measured at a single point after the onset of PCOS may not represent continuous levels of exposure before onset of diseases in the current study.

- The inclusion of infertility women with genital tract inflammation in the control group may have drawn the estimates towards the null.

- As with all cross-sectional studies, it is uncertain whether TCS exposure preceded PCOS. Therefore, a causal relationship cannot be assured based on the current study.

- TCS exposure was not measured in a substantial proportion of the original study population, limiting the generalisability of the results.

was observed in about $60 \%$ of women with PCOS, ${ }^{1}{ }^{2}$ whereas the elevated $\mathrm{LH} /$ follicle stimulate hormone (FSH) ratio could be found in $95 \%$ of PCOS cases. ${ }^{3}$ Although the aetiology of the syndrome remained unclear, potential effects of environmental endocrine-disrupting compounds (EEDCs) on the development of PCOS have drawn increasing attention. $^{45}$ For example, animal studies have demonstrated that bisphenol A (BPA), a widely recognised EEDC, is involved in the pathogenesis of PCOS. ${ }^{4}$ BPA has also been found to be associated with reduced female fertility ${ }^{6}$ and reduced anogenital distance in male infants. ${ }^{7}$

Triclosan (TCS) is a chlorinated aromatic compound that has a similar chemical structure to BPA. It is widely used in personal care as well as household products such as perfumed soaps, shampoo, toothpaste and liquid disinfectants. ${ }^{8-10}$ Widespread exposures to TCS have been observed in many countries. ${ }^{11-14}$ Public concerns over the ubiquitous exposure 
to TCS and other EEDCs have increased. ${ }^{12-15}$ Evidence from animal and in vitro studies suggested a variety of hormonal activities of TCS, including oestrogenic, androgenic and antiandrogenic activities, and disturbance of thyroid hormone activities, ${ }^{16-19}$ all of which are related to PCOS-like syndrome. But human studies are few.

Based on the structure-activity relationship, we hypothesise that like BPA, TCS may mimic, alter or attenuate oestradiol and other natural hormone functions. ${ }^{16} \mathrm{We}$ speculate that effects of TCS exposure on reproductive hormones may result in PCOS symptoms such as hyperandrogenism, anovulation and elevated LH. For example, inverse associations were found between TCS exposure and pubic hair development in girls, ${ }^{14}$ anogenital distance at 3 months of age in boys ${ }^{12}$ and head circumference at birth. ${ }^{11}$ Although results from human studies were often inconsistent, the available evidence suggests that TCS exposure may affect the hypothalamic-pituitary-gonadal axis (HPGA) and lead to changes in production and action of reproductive hormones. Thus, we speculated that TCS exposure may contribute to the pathogenesis of PCOS. The aim of the current study is to examine the association between TCS and PCOS, and explore possible mechanisms on how TCS may contribute to the development of clinical manifestation of PCOS.

\section{METHODS}

\section{Study sample}

All infertile women at 18-45 years of age who came to the Women's Hospital, Zhejiang University School of Medicine for infertility were potentially eligible for the current study. From March 2014 to August 2015, a total of 705 infertile women were recruited. Infertility cases due to genital tract malformation with or without surgery $(n=31)$ were excluded from this study. Infertility due to PCOS was treated as the case of interest while male infertility (women presenting for artificial insemination with donor's semen (AID) or intracytoplasmic sperm injection (ICSI)) or genital tract infection, tubal obstruction or adhesion but with a normal sex hormone profile were taken as the control group. PCOS was diagnosed according to the revised 2003 Rotterdam criteria.

A total of 118 PCOS cases and 556 non-PCOS infertile women were selected. We limited the analyses to 84 PCOS cases and 212 controls who had urinary TCS measured. Among the 212 controls, 74 were due to male infertility for AID or ICSI and 138 diagnosed of infertility due to genital tract infection, tubal obstruction or adhesion but with a normal sex hormone profile (figure 1).

An in-person interview was administered by a trained research assistant to collect information on women's demographic, environmental exposure and behavioural characteristics. Clinical information (eg, reproductive history and baseline reproductive hormones levels) was abstracted from the medical record. Blood and urine samples were also collected on the same day as the questionnaire was administered.

Urine specimen collection and TCS measurements.

About $100 \mathrm{~mL}$ urine samples were collected in sterile polypropylene cups at the visit. Samples were aliquoted to polypropylene storage tubes $(15 \mathrm{~mL}, 430791$ CentriStar, Coring, California, USA) and stored at $-20^{\circ} \mathrm{C}$ prior to

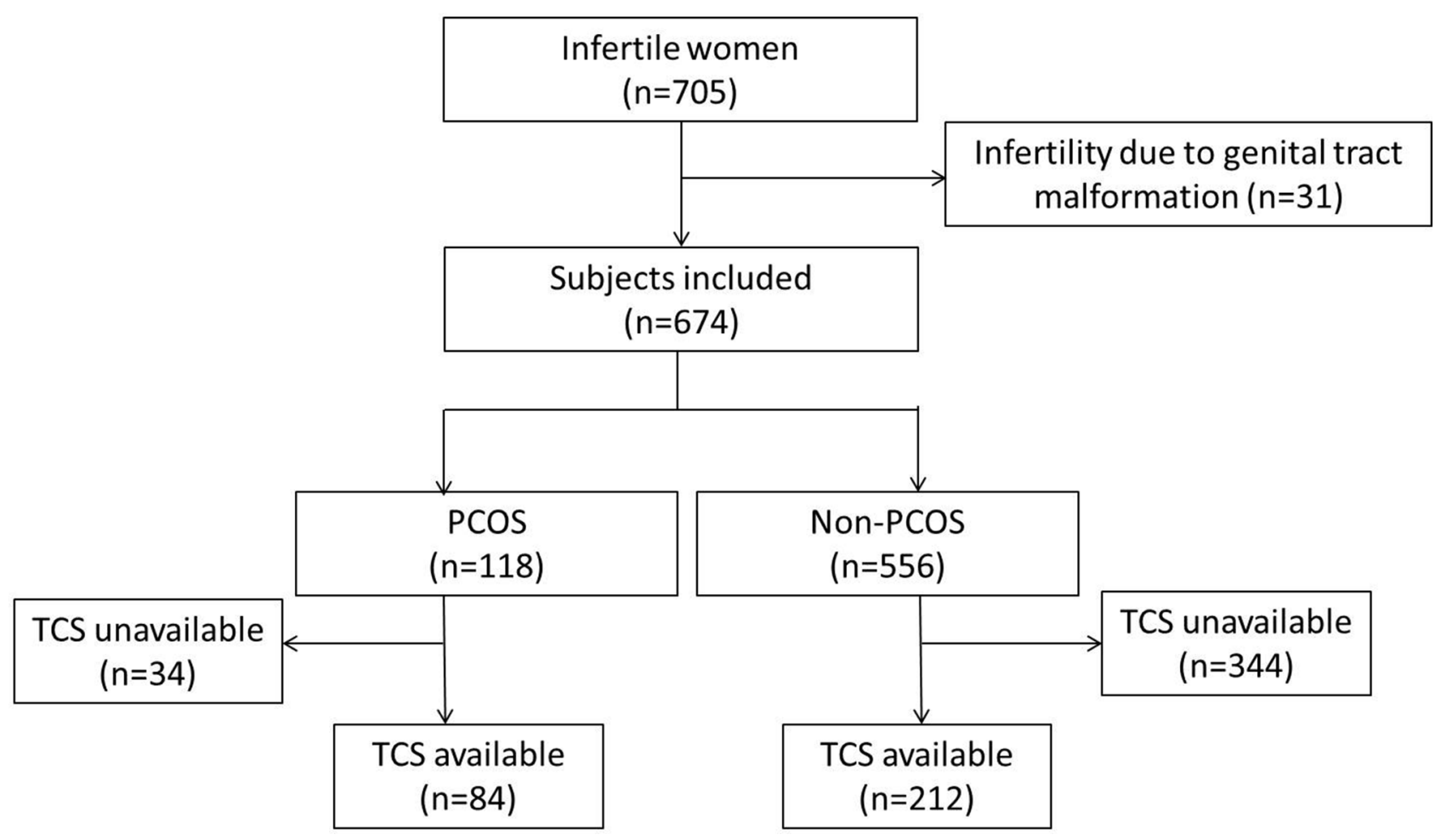

Figure 1 Study subjects flow chart. PCOS, polycystic ovary syndrome; TCS, triclosan. 
TCS analysis. Urinary TCS concentration was measured using high-performance liquid chromatography-electrospray ionisation tandem mass spectrometry (Agilent 1290-64090, Agilent Technologies, Little Falls, Delaware, USA). The limit of detection (LOD) was $0.1 \mathrm{ng} / \mathrm{mL}$. TCS concentrations were corrected for urine dilution by creatinine. Creatinine concentration was measured using enzymatic method on automatic chemical analyser (7100 Automatic Analyzer, Hitachi, Tokyo, Japan). More details on urinary TCS analytical methods were provided elsewhere. ${ }^{20}$ We substituted (LOD) $/ 2$ for the results below LOD.

\section{Outcomes}

PCOS cases were diagnosed based on the revised 2003 Rotterdam criteria, in which a woman meets at least two out of three criteria: oligo-ovulation or anovulation; clinical and/or biochemical signs of hyperandrogenism; polycystic ovaries and exclusion of other aetiologies (congenital adrenal hyperplasia, androgen-secreting tumours, Cushing's syndrome). ${ }^{1}$ Levels of oestradiol, progesterone, LH and FSH were measured in serum collected on the second or third day of the menstrual cycle, using an automated electrochemiluminescence immunoassay.

\section{Data analysis}

Normally distributed variables were presented as means and SD. The two-tailed unpaired t-test was used to evaluate the difference between PCOS and non-PCOS group. Variables with skewed distribution were described as median and IQR. Wilcoxon two-sample test was used to assess the difference in variables with a skewed distribution between two groups. TCS was grouped by tertiles and included as an ordered categorical variable in the logistic regression models for the association between TCS and PCOS. Potential covariates included age, education, smoking, alcohol intake, caffeine consumption, body mass index (BMI). ${ }^{12}$ We conducted a multiple imputation (five imputations) using the Markov chain Monte Carlo method for the missing values of smoking, alcohol and caffeine consumption. ${ }^{21} 22$ Then we explored the relationship of PCOS with smoking, drinking and caffeine consumption in crude and adjusted models. None of these variables was significantly associated with the outcome $(p<0.05)$ using either imputed or the original database. Therefore, they were not retained in the final models. Finally, age, education and BMI were entered into the multivariable models.

To explore the potential non-linear association of TCS exposure with PCOS, fractional polynomial regression models were used to fit the relationship between urinary TCS levels and LH, FSH, LH/FSH ratio, oestradiol and progesterone. As an extension of polynomial regression models, the power terms of TCS concentration are chosen from a predefined set of values $(-2,-1,-0.5,0$, $0.5,1,2,3)$. Since the first or second degree of fractional polynomials can provide adequate flexibility, we chose the best model from first or second degree of fractional polynomials without consideration a higher degree model. The best fit model is defined as the one that maximises the likelihood (minimises the deviance). ${ }^{23}{ }^{24}$ The alternative models and deviances for the relationships between TCS and LH, LH/FSH and oestradiol were shown in online supplementary appendix tables 1 and 2. Deviance residuals plotted against urinary TCS concentrations showed no non-random trends or patterns for the selected models. The significance tests based on the comparison of deviance among best fitted first degree, second degree polynomial models, linear models and models without any power term of TCS concentration were presented in online supplementary appendix tables 3 and 4 and supplementary appendix figures 1 and 2 . Because the power term of TCS concentration for the best fitted first degree model was not nested within that of the best fitted second degree model, the comparison between

Table 1 Descriptive statistics of the characteristics of women with and without polycystic ovary syndrome (PCOS)

\begin{tabular}{|c|c|c|c|}
\hline & $\begin{array}{l}\text { PCOS } \\
(n=84)\end{array}$ & $\begin{array}{l}\text { Non-PCOS } \\
(n=212)\end{array}$ & $P$ values \\
\hline Age (mean (SD), year) & $30.4(3.6)$ & $32.2(4.2)$ & 0.0012 \\
\hline $\begin{array}{l}\text { Body mass index ((mean } \\
\left.(\mathrm{SD}), \mathrm{kg} / \mathrm{m}^{2}\right)\end{array}$ & $21.9(3.2)$ & $22.0(3.6)$ & 0.8067 \\
\hline Overweight (\%) & 18.1 & 16.3 & 0.7091 \\
\hline $\begin{array}{l}\text { Age at menarche (mean } \\
\text { (SD), year) }\end{array}$ & $14.0(1.4)$ & $14.3(1.8)$ & 0.0874 \\
\hline $\begin{array}{l}\text { Menstrual cycle (mean } \\
\text { (SD), day) }\end{array}$ & $30.2(5.9)$ & $29.8(5.1)$ & 0.6183 \\
\hline
\end{tabular}

Education (\%)

\begin{tabular}{|c|c|c|c|}
\hline $\begin{array}{l}\text { Primary school and } \\
\text { below }\end{array}$ & 3 (3.6) & $16(7.6)$ & $<0.0001$ \\
\hline $\begin{array}{l}\text { Junior and senior high } \\
\text { school }\end{array}$ & 34 (40.5) & $135(63.7)$ & \\
\hline $\begin{array}{l}\text { College, university } \\
\text { and postgraduate }\end{array}$ & $44(52.4)$ & $51(24.1)$ & \\
\hline Missing & 3 (3.6) & $10(4.7)$ & \\
\hline Smoking (\%) & & & 0.3721 \\
\hline No & 67 (79.8) & $154(72.6)$ & \\
\hline Yes & $2(2.4)$ & $4(1.9)$ & \\
\hline Missing & 15 (17.9) & $54(25.5)$ & \\
\hline Alcohol (\%) & & & 0.6644 \\
\hline No & $26(31.0)$ & 71 (33.5) & \\
\hline Occasionally & 33 (39.3) & 80 (37.7) & \\
\hline Often & $12(14.3)$ & 21 (9.9) & \\
\hline Missing & 13 (15.5) & 40 (18.9) & \\
\hline Caffeine (\%) & & & 0.2101 \\
\hline Non & 49 (58.3) & $126(59.4)$ & \\
\hline Occasionally & $23(27.4)$ & $43(20.3)$ & \\
\hline Often & $4(4.8)$ & $6(2.8)$ & \\
\hline Missing & $8(9.5)$ & 37 (17.5) & \\
\hline
\end{tabular}


Table 2 Descriptive statistics of TCS, LH, FSH, oestradiol and progesterone in women with and without PCOS

\begin{tabular}{llll}
\hline & PCOS (n=84) & Non-PCOS (n=212) & P values \\
\hline TCS (median (IQR), ng/mL) & $0.45(0.25-1.54)$ & $0.37(0.14-0.98)$ & 0.0249 \\
TCS_Cre* (median (IQR), $\mu \mathrm{g} / \mathrm{g}$ creatinine) & $1.49(0.68-3.80)$ & $1.06(0.52-3.02)$ & 0.0407 \\
LH (mean (SD), IU/L) & $9.22(6.05),(40)$ & $7.81(7.04),(125)$ & 0.2552 \\
FSH (mean (SD), IU/L) & $5.97(2.03),(40)$ & $6.57(1.87),(124)$ & 0.0865 \\
LH/FSH (mean (SD)) & $1.65(1.05),(40)$ & $1.22(0.85),(124)$ & 0.0094 \\
Oestradiol (median (IQR), pmol/L) & $127.96(88.55-229.49),(40)$ & $182.90(116.90-311.28),(125)$ & 0.0164 \\
Progesterone (median (IQR), ng/mL) & $1.73(1.16-2.17)$ & $1.85(1.26-2.49),(122)$ & 0.2625 \\
& $(39)$ & & \\
\hline
\end{tabular}

*TCS_Cre, creatinine-corrected TCS concentration.

$\mathrm{FSH}$, follicle stimulate hormone; LH, luteinising hormone; PCOS, polycystic ovary syndrome; TCS, triclosan.

models based on the deviances only gives approximate $\mathrm{p}$ values. The model with the minimal deviance was chosen as the best fitted model.

The analyses of associations between TCS and reproductive hormones levels were restricted to non-PCOS participants to avoid potential interferences of PCOS. The hormone levels were log-transformed wherever appropriate. Serum level of oestradiol was $\log 10$-transformed because of its skewed distribution. We first fitted crude fractional polynomial regression models and then explored adjusted models. All covariates retained in the aforementioned logistic regression models were retained in this analysis. We further adjusted oestradiol to evaluate the independent effects of TCS on the development of symptoms of PCOS by controlling feedback regulations of oestradiol on LH and FSH. All analyses were carried out using the SAS V.9.2 (SAS Institute).

\section{PATIENT AND PUBLIC INVOLVEMENT STATEMENT}

The patients and public were not involved in the study design or setting the research agenda.

\section{RESULTS}

We first compared women who had TCS measurements and were included in the final analyses with those who did not have TCS measurements. There were no significant differences in demographic characteristics or reproductive hormones levels between these two groups except

\begin{tabular}{|c|c|c|c|}
\hline TCS & $\mathbf{n}$ & $\begin{array}{l}\text { Crude OR } \\
(95 \% \mathrm{Cl})^{*}\end{array}$ & $\begin{array}{l}\text { Adjusted OR } \\
(95 \% \mathrm{Cl}) \dagger\end{array}$ \\
\hline$<0.68$ & 101 & Reference & Reference \\
\hline $0.68-2.21$ & 97 & 1.55 (0.81 to 2.96$)$ & 1.47 (0.76 to 2.84$)$ \\
\hline$\geq 2.21$ & 98 & 2.12 (1.12 to 3.99 ) & 1.99 (1.05 to 3.79$)$ \\
\hline
\end{tabular}

${ }^{*} P$ trend $_{\text {crude }}=0.0204$.

†Adjusted for age, education and BMI; $p$ trend ${ }_{\text {adjusted }}=0.0351$. BMI, body mass index; PCOS, polycystic ovary syndrome; TCS, triclosan. for education level. But subjects without TCS measurements had more missing values of tobacco, alcohol and caffeine consumption. After the multiple imputations for missing values, only alcohol consumption was significantly different between women who had and did not have TCS measurements in the control group (online supplementary appendix tables 5 and 6). A total of 84 infertile women with PCOS and 212 women without PCOS were retained in the analyses. The mean age of participants was 31.7 (SD 4.1). Women in the PCOS group were younger and had higher education levels than those of the non-PCOS group (table 1). There were no significant differences between the two groups in tobacco, alcohol and caffeine consumption. Eighteen per cent and $16 \%$ of the women were overweight or obese in these two groups, respectively. Age at menarche and menstrual cycle length were similar ( $\mathrm{p}=0.0874$ and 0.6138 , respectively, table 1$)$.

Urinary TCS concentration was above the LOD in $90.5 \%(76 / 84)$ and $83.7 \%(185 / 221)$ of PCOS cases and controls, respectively. The results below LOD were substituted by (LOD)/2. The PCOS group had significantly higher TCS concentrations (with or without correction for creatinine) than the control group (table 2). Serum LH level was higher in the cases than the controls, but the difference was not statistically significant. The LH/ FSH ratio was elevated significantly in the PCOS group as compared with the non-PCOS group. Decreased oestradiol concentration was observed in the PCOS group. There is no significantly difference in serum FSH or progesterone between the two groups (table 2).

The creatinine-corrected TCS levels were divided into three tertiles. We fitted a logistic regression model for the relationship between creatinine-corrected TCS levels and the probability of PCOS. Higher TCS level was associated with an increased odd of PCOS ( $p$ for trend=0.0204). The significantly increased OR of PCOS was showed in the highest tertile when compared with the lowest tertile (OR 2.12, 95\% CI 1.12 to 3.99). After adjusting for covariates, the significant association remained (OR 1.99, 95\% CI 1.05 to 3.79) (table 3).

To explore the association between TCS exposure and the developments of PCOS-associated symptoms, 

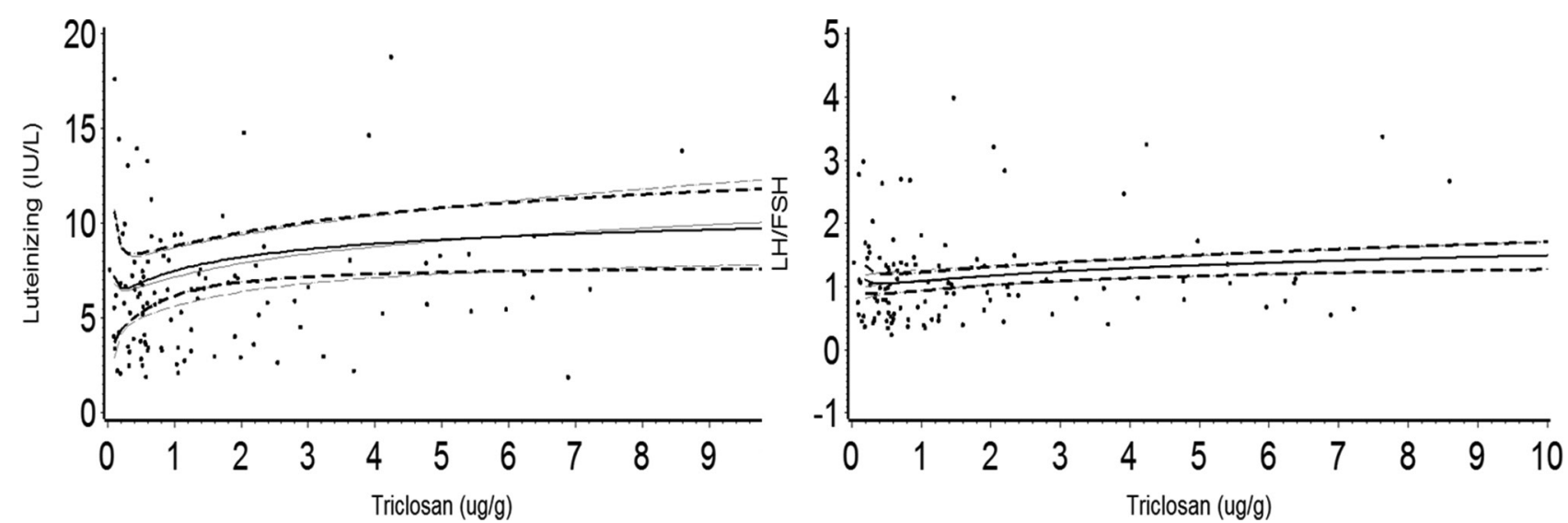

Figure 2 Dot plots and predicted curves based on fractional polynomial models fit for the association between TCS and LH and LH/FSH. Grey curves: predicted curve (solid curve) and 95\% Cl (dashed curves) fit to fractional polynomial models adjusting for age, education and BMI. Black curves: predicted curve (solid curve) and 95\% $\mathrm{Cl}$ (dashed curves) fit to fractional polynomial models adjusting for age, education, BMI and oestradiol. BMI, body mass index; FSH, follicle stimulate hormone; $\mathrm{LH}$, luteinising hormone; TCS, triclosan.

we limited the further analyses to non-PCOS participants. We fitted fractional polynomial regression models to explore the possible non-linear associations between creatinine-corrected TCS and LH, FSH, LH/FSH, oestradiol and progesterone. Non-linear association was found between urinary levels of TCS and LH in both crude and adjusted models (online supplementary appendix table 7). Even after adjusting for oestradiol, trends of the curves were essentially unchanged (figure 2). The fitted curves showed that LH increased gradually with elevated TCS when TCS level was below $1-2 \mathrm{ng} / \mathrm{g}$. But LH level plateaued when TCS concentration increased further. The predicted values and 95\% CI of LH and LH/ FSH based on the selected models were presented in online supplementary appendix table 8 .

Non-linear associations were also found between TCS and LH/FSH ratio (online supplementary appendix tables 7 and 8). As depicted in figure 2, LH/FSH ratio increased slightly with the elevated levels of TCS. The crude and adjusted models (with and without adjusting for oestradiol) also generated similar results (red, blue and black curves, online supplementary appendix figure $3)$.

\section{DISCUSSION}

Our study showed that infertile women with PCOS had a significantly higher TCS level than infertile women without PCOS. Non-linear relationships were found between TCS concentrations and $\mathrm{LH}$ and $\mathrm{LH} / \mathrm{FSH}$ ratio in women without PCOS. These findings suggested that TCS may be associated with the developments of PCOS.

The urinary TCS level in our study population (1.49 (IQR: 0.68-3.80) and 1.06 (IQR: 0.52-3.02) $\mu \mathrm{g} / \mathrm{g}$ in the PCOS and non-PCOS group, respectively) was somewhat lower than that reported in Danish women (geometric mean of TCS $=1.01 \mathrm{ng} / \mathrm{mL}),{ }^{12}$ and much lower than that in girls in the USA (geometric mean of TCS $=12.4 \mu \mathrm{g} / \mathrm{g}$ creatinine) based on the data from the Centers for Disease
Control and Prevention's National Health and Nutrition Examination Survey. ${ }^{13}$ But it was compatible with serum concentrations of TCS at 0.549 and $0.649 \mathrm{ng} / \mathrm{mL}$ in pregnant women with normal and abnormal fetuses reported in a previous study in Beijing, China. ${ }^{11}$ In a another study in China (1997-2000), the creatinine-corrected TCS level (median TCS concentration was under the LOD $2.3 \mu \mathrm{g} / \mathrm{L})^{3}$ was also much lower than that in the USA and Europe. Human TCS exposure mostly came from common personal care and household products such as perfume soap, shampoo, toothpaste and liquid detergent. TCS is also widely added to personal care and household products as an antibacterial and antifungal agent in China, but the personal care products may not be used as frequently as that in the USA and Europe. For example, deodorant is composed of a high concentration of TCS. Women in China rarely use deodorant. This may not be the case in some Western countries. Although it would be difficult to compare the contact frequency with personal care and household products across studies without standardised questionnaire, we may speculate that different TCS exposure levels may be due to different consumer behaviour. We found a positive association between urinary TCS and the contact frequency with perfume soap/shampoo and liquid detergent (data not shown). The difference in TCS levels across studies may reflect differences in consumer behaviour of personal care and household products among countries and regions.

A variety of reproductive hormone-like actions exerted by TCS have been reported in vitro and animal experiments, including oestrogenic, androgenic and antiandrogenic effects. ${ }^{12} 171825$ Although different mechanisms as well as high tissue/organ specificity that TCS has may impact dose-dependent responses, ${ }^{26}$ ample evidence indicated that TCS may have a wide range of actions through its effects on HPGA. ${ }^{12}$ In contrast to the reduced LH and FSH concentrations found to be associated with an increased TCS levels in an animal experiment in which 
rats were treated with TCS at a high-dose $(20 \mathrm{mg} / \mathrm{kg}$ body weight per day for a period of 60 days by intubation), ${ }^{27}$ the positive association between TCS and LH concentration and LH/FSH ratio found in a low-dose exposed population like ours was compatible to the findings in other previous studies. ${ }^{162}$ Different species, doses, duration, timing and route of exposure may lead to diverse modes of actions for TCS in vitro, in animal and epidemiological studies.

Elevated LH concentrations and LH/FSH ratio were common clinical manifestation of patients with PCOS. ${ }^{1-329}$ The positive association between TCS and LH and LH/ FSH ratio in non-PCOS group suggested that TCS may contribute to the pathogenesis of PCOS. TCS at high and low dose may have different reproductive hormonelike actions. Even though humans are exposed to TCS at much lower level than that of the animal models, TCS still shows endocrine disrupting effects.

\section{Strength and limitations}

To our knowledge, this is the first study that provides evidence that TCS may play a role in the development of PCOS in human. However, several limitations are worth mentioning. First, the present study was a cross-sectional investigation. TCS levels were measured at a single point after the onset of PCOS. The concentration may not represent continuous levels of exposure before the onset of the disease. Ideally, we should measure urinary TCS several times in a prospective cohort study. However, a person's lifestyle usually does not change dramatically over time; exposure to environmental TCS is likely to be more or less stable. Thus, the spot urinary TCS would reflect a general exposure level of individual. ${ }^{30}$ Second, the comparison group in this study consisted of infertility women due to genital tract inflammation and male infertility. TCS may be associated with both endocrine function and inflammation reaction. ${ }^{16}{ }^{31}$ The inclusion of infertility women with genital tract inflammation may have drawn the estimates towards the null. But in a sensitivity analysis, we found no significant association of TCS with genital inflammation. Neither did we find remarkable changes in the relationships between TCS and oestradiol, LH and FSH after adjusting for inflammation (data not shown). Third, effects of EEDCs are rarely uniform across the entire dose-response range. ${ }^{26}$ The whole range of TCS exposure in the current study was at relatively low levels. We cannot extrapolate reproductive effects of TCS beyond the available range in the current study. Thus, our findings of the positive non-linear association between TCS and symptoms of PCOS (elevated LH and LH/FSH ratio) may not be generalised to a population of higher exposure. Fourth, although all subjects were given a full workup for the diagnosis of PCOS, the misclassification of PCOS and non-PCOS may exist. Since this misclassification was not associated with TCS exposure level, the bias was likely to have drawn the results towards the null. Fifth, the study subjects are volunteers from the infertility clinic. It may not totally represent all the infertile patients. However, it is unlikely that the self-selection bias was based on the level of TCS exposure. This limitation, therefore, may reduce the generalisability of the results to other population but not necessarily the internal validity of the findings. Finally, although unique, the sample size of the current study is still small. Larger studies are needed to confirm or refute our finding.

\section{CONCLUSIONS}

TCS exposure was significantly associated with PCOS in a Chinese population. $\mathrm{LH}$ and $\mathrm{LH} / \mathrm{FSH}$ ratio increased non-linearly with elevated TCS concentration at a relatively low level in women with a normal reproductive endocrine function. Further epidemiological studies are needed to address this important, modifiable issue that may have important public health implications.

\section{Author affiliations}

${ }^{1}$ Department of Clinical Epidemiology, Institute of Obstetrics and Gynecology, Obstetrics and Gynecology Hospital, Fudan University, Shanghai, China

${ }^{2}$ Department of Reproductive Endocrinology, Key Laboratory of Reproductive Genetics of Ministry of Education (Zhejiang University), Key Laboratory of Women's Reproductive Health of Zhejiang Province, Women's Hospital, Zhejiang University School of Medicine, Hangzhou, Zhejiang, China

${ }^{3}$ Ministry of Education-Shanghai Key Laboratory of Children's Environmental Health, Xinhua Hospital, Shanghai Jiao Tong University School of Medicine, Shanghai, China ${ }^{4}$ Key Laboratory of Women's Reproductive Health of Zhejiang Province, Women's Hospital, Zhejiang University School of Medicine, Hangzhou, Zhejiang, China

${ }^{5}$ School of Public Health, Guilin Medical School, Guilin, Guangxi, China

Contributors JZ and FJ conceived this study. JY analysed the data and drafted the manuscript. JZ supervised the analysis, provided guidance to data analysis and interpretation, and provided critical feedback on manuscript drafts. FJ, WZ, HL and YM provided critical feedback on interpretation of results and on the manuscript draft.

Funding This work was supported by the Ministry of Science and Technology of China (grant number 2014CB943300).

Competing interests None declared.

Patient consent Obtained.

Ethics approval The study protocol was approved by the Ethics Committees of the Women's Hospital, Zhejiang University School of Medicine and Xinhua Hospital, Shanghai Jiao Tong University School of Medicine.

Provenance and peer review Not commissioned; externally peer reviewed.

Data sharing statement № additional data are available.

Open access This is an open access article distributed in accordance with the Creative Commons Attribution Non Commercial (CC BY-NC 4.0) license, which permits others to distribute, remix, adapt, build upon this work non-commercially, and license their derivative works on different terms, provided the original work is properly cited, appropriate credit is given, any changes made indicated, and the use is non-commercial. See: http://creativecommons.org/licenses/by-nc/4.0/.

\section{REFERENCES}

1. Rotterdam ESHRE/ASRM-Sponsored PCOS Consensus Workshop Group. Revised 2003 consensus on diagnostic criteria and longterm health risks related to polycystic ovary syndrome. Fertil Steril 2004;81:19-25.

2. Laven JS, Imani B, Eijkemans MJ, et al. New approach to polycystic ovary syndrome and other forms of anovulatory infertility. Obstet Gynecol Surv 2002;57:755-67.

3. Taylor AE, McCourt B, Martin KA, et al. Determinants of abnormal gonadotropin secretion in clinically defined women with polycystic ovary syndrome. J Clin Endocrinol Metab 1997;82:2248-56. 
4. Kandaraki E, Chatzigeorgiou A, Livadas S, et al. Endocrine disruptors and polycystic ovary syndrome (PCOS): elevated serum levels of bisphenol A in women with PCOS. J Clin Endocrinol Metab 2011;96:E480-E484.

5. Diamanti-Kandarakis E, Katsikis I, Piperi C, et al. Increased serum advanced glycation end-products is a distinct finding in lean women with polycystic ovary syndrome (PCOS). Clin Endocrinol 2008;69:634-41.

6. Gray LE, Laskey J, Ostby J. Chronic di-n-butyl phthalate exposure in rats reduces fertility and alters ovarian function during pregnancy in female Long Evans hooded rats. Toxicol Sci 2006;93:189-95.

7. Bornehag CG, Carlstedt F, Jönsson BA, et al. Prenatal phthalate exposures and anogenital distance in Swedish boys. Environ Health Perspect 2015;123:101-7.

8. Casas L, Fernández MF, Llop S, et al. Urinary concentrations of phthalates and phenols in a population of Spanish pregnant women and children. Environ Int 2011;37:858-66.

9. Philippat C, Botton J, Calafat AM, et al. Prenatal exposure to phenols and growth in boys. Epidemiology 2014;25:625-35.

10. Wolff MS, Engel SM, Berkowitz GS, et al. Prenatal phenol and phthalate exposures and birth outcomes. Environ Health Perspect 2008;116:1092-7.

11. Wei L, Qiao P, Shi Y, et al. Triclosan/triclocarban levels in maternal and umbilical blood samples and their association with fetal malformation. Clinica Chimica Acta 2017;466:133-7.

12. Lassen $\mathrm{TH}$, Frederiksen $\mathrm{H}$, Kyhl HB, et al. Prenatal triclosan exposure and anthropometric measures including anogenital distance in danish infants. Environ Health Perspect 2016:124:1261-8.

13. Buttke DE, Sircar K, Martin C. Exposures to endocrine-disrupting chemicals and age of menarche in adolescent girls in NHANES (2003-2008). Environ Health Perspect 2012;120:1613-8.

14. Wolff MS, Teitelbaum SL, Pinney SM, et al. Investigation of relationships between urinary biomarkers of phytoestrogens, phthalates, and phenols and pubertal stages in girls. Environ Health Perspect 2010;118:1039-46.

15. Jukic AM, Calafat AM, McConnaughey DR, et al. Urinary concentrations of phthalate metabolites and bisphenol a and associations with follicular-phase length, luteal-phase length, fecundability, and early pregnancy loss. Environ Health Perspect 2016;124:321-8.

16. Ahn KC, Zhao B, Chen J, et al. In vitro biologic activities of the antimicrobials triclocarban, its analogs, and triclosan in bioassay screens: receptor-based bioassay screens. Environ Health Perspect 2008;116:1203-10.
17. Christen V, Crettaz P, Oberli-Schrämmli A, et al. Some flame retardants and the antimicrobials triclosan and triclocarban enhance the androgenic activity in vitro. Chemosphere 2010;81:1245-52.

18. Gee RH, Charles A, Taylor N, et al. Oestrogenic and androgenic activity of triclosan in breast cancer cells. Journal of Applied Toxicology 2008;28:78-91.

19. Axelstad M, Boberg J, Vinggaard AM, et al. Triclosan exposure reduces thyroxine levels in pregnant and lactating rat dams and in directly exposed offspring. Food and Chemical Toxicology 2013:59:534-40.

20. Zhao S, Zhang $\mathrm{H}$, Zhang J, et al. Establishment and application of determination method for BPA, TCS and 4-N-NP in urine by HPLCMS/MS. J. Environ. Occup. Med 2015;32:846-51.

21. Schafer JL. Multiple imputation: a primer. Stat Methods Med Res 1999;8:3-15.

22. SAS Institute Inc. SAS/STAT 9 User's Guide. North Carolina: SAS Institute Inc, 2003

23. Royston P, Sauerbrei W. Building multivariable regression models with continuous covariates in clinical epidemiology-with an emphasis on fractional polynomials. Methods of information in medicine 2005;44:561-71.

24. Royston P, Ambler G, Sauerbrei W. The use of fractional polynomials to model continuous risk variables in epidemiology. Int $\mathrm{J}$ Epidemiol 1999;28:964-74.

25. Schiffer C, Muller A, Egeberg DL, et al. Direct action of endocrine disrupting chemicals on human sperm. EMBO Rep 2014:15:758-65.

26. Gennings C, Carter WH, Carney EW, et al. A novel flexible approach for evaluating fixed ratio mixtures of full and partial agonists. Toxicol Sci 2004;80:134-50.

27. Kumar V, Chakraborty A, Kural MR, et al. Alteration of testicular steroidogenesis and histopathology of reproductive system in male rats treated with triclosan. Reprod Toxicol 2009;27:177-85.

28. Stoker TE, Gibson EK, Zorrilla LM. Triclosan exposure modulates estrogen-dependent responses in the female wistar rat. Toxicol Sci 2010;117:45-53.

29. van Santbrink EJP, Hop WC, Fauser BCJM. Classification of normogonadotropic infertility: polycystic ovaries diagnosed by ultrasound versus endocrine characteristics of polycystic ovary syndrome. Fertil Steril 1997;67:452-8.

30. Lassen TH, Frederiksen $\mathrm{H}$, Jensen TK, et al. Temporal variability in urinary excretion of bisphenol $A$ and seven other phenols in spot, morning, and 24-h urine samples. Environ Res 2013;126:164-70.

31. Ashley-Martin J, Dodds L, Arbuckle TE, et al. Prenatal triclosan exposure and cord blood immune system biomarkers. Int $\mathrm{J}$ Hyg Environ Health 2016;219(4-5):454-7. 